\title{
Finding Hidden Quasars with UKIDSS and AAOmega
}

\author{
Natasha Maddox $^{1}$, P. C. Hewett ${ }^{1}$, S. J. Warren ${ }^{2}$ and S. M. Croom ${ }^{3}$ \\ ${ }^{1}$ Institute of Astronomy, University of Cambridge, Madingley Road, Cambridge, \\ CB3 0HA, UK \\ email: nmaddox@ast.cam.ac.uk \\ ${ }^{2}$ Astrophysics Group, Imperial College London, Blackett Laboratory, Prince Consort Road, \\ London, SW7 2AZ, UK \\ ${ }^{3}$ School of Physics, University of Sydney, NSW, 2006, Australia
}

The debate regarding the number of dust-reddened quasars has been ongoing for several years. Selecting quasars based on a UV excess will exclude any quasar that has an SED that departs from the standard power-law or is experiencing obscuration from dust. As longer wavelengths are less affected by dust attenuation, it is advantageous to select quasar candidates in the NIR, but until now, there did not exist a dataset of sufficient depth and coverage suitable for undertaking such an experiment.

UKIDSS (Lawrence et al. 2006) is a set of five separate surveys with complementary combinations of coverage and depth. The Large Area Survey (LAS) is the largest of the surveys, and will cover $4000 \mathrm{deg}^{2}$ to $K=18.2$ in the SDSS footprint over 7 years. The LAS provides the large area, high quality data required for creating a $K$-band selected quasar sample. Coupling with existing SDSS data provides ugrizYJHK photometry plus morphology information for each detected object.

Analogous to the UV-excess seen at shorter wavelengths, there is an equivalent excess in the $K$-band for quasars compared to stars (KX; Warren, Hewett \& Foltz 2000). This may be exploited by combining optical-NIR colours, for example $g J K$, in a two colour plot. The stars are confined to a well-defined locus, clearly separated from the quasars and galaxies. The technique is reddening-insensitive, as dust tends to move quasars further from the stellar locus. Spectroscopic observations of the quasar candidates were under-

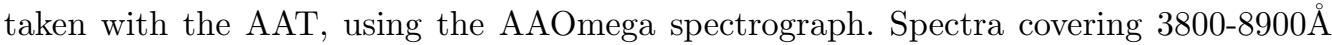
over $16.5 \operatorname{deg}^{2}$ confirmed more than 200 quasars to $K<17$, with redshifts ranging from $0.27<z<3.7$.

The redshift distribution of KX-confirmed quasars shows a large peak at low redshifts, indicating the importance of host galaxy flux when selecting at longer wavelengths, consistent with predictions made in Maddox \& Hewett (2006). There is also no significant lack of quasars between $2<z<3$ as is apparent in optically selected samples. Inspection of the confirmed quasars reveals a significant population of objects that satisfy the SDSS $i$-band magnitude limits for quasar target selection but have no SDSS spectroscopic data. This issue is particularly evident at $z>3$, where 7 quasars were confirmed in this study but only two quasars were confirmed by the SDSS.

\section{References}

Lawrence, A. et al. 2006, MNRAS, submitted, astro-ph/0604426

Maddox, N. \& Hewett, P.C. 2006, MNRAS 367, 717

Warren, S.J., Hewett, P.C. \& Foltz, C.B. 2000, MNRAS 312, 827 